\title{
Uniform Noting for International Application of the Tumor-Stroma Ratio as an Easy Diagnostic Tool: Protocol for a Multicenter Prospective Cohort Study
}

Marloes Smit ${ }^{1}$, MD; Gabi van Pelt ${ }^{1}$, BSc; Annet Roodvoets ${ }^{2}$, MSc; Elma Meershoek-Klein Kranenbarg ${ }^{2}$, MSc; Hein Putter $^{3}$, MSc, PhD; Rob Tollenaar ${ }^{1}, \mathrm{MD}, \mathrm{PhD} ; \mathrm{J}_{\text {Han van Krieken }}^{4}$, MD, PhD; Wilma Mesker ${ }^{1}, \mathrm{PhD}$

${ }^{1}$ Department of Surgery, Leiden University Medical Center, Leiden, Netherlands

${ }^{2}$ Datacenter Surgery, Leiden University Medical Center, Leiden, Netherlands

${ }^{3}$ Department of Medical Statistics, Leiden University Medical Center, Leiden, Netherlands

${ }^{4}$ Department of Pathology, Radboud University Medical Center, Nijmegen, Netherlands

\section{Corresponding Author:}

Wilma Mesker, $\mathrm{PhD}$

Department of Surgery

Leiden University Medical Center

Albinusdreef 2

Leiden, $2333 \mathrm{ZA}$

Netherlands

Phone: 31715264005

Email: W.E.Mesker@lumc.nl

\section{Abstract}

Background: Colon cancer treatment is dependent on the stage at diagnosis. The current Tumor-Node-Metastasis (TNM) staging for the selection of patients for adjuvant chemotherapy needs additional prognostic and predictive biomarkers. Better decision making for chemotherapy will result in reducing over- and undertreatment. We developed a new, easy-to-apply, practice-changing method to select colon cancer patients for adjuvant chemotherapy: the tumor-stroma ratio (TSR). The TSR distinguishes within stage II-III patients who will likely benefit from adjuvant chemotherapy and those who will not.

Objective: The aim of the study was to add, in addition to the TNM classification, the TSR to current routine pathology evaluation. Pathologists will be instructed for scoring the TSR in combination with a quality assessment program. An international multicenter study will validate the parameter prospectively.

Methods: The study is designed for future implementation of the TSR to the current TNM guidelines, using routinely Haematoxylin- and Eosin-stained tumor tissue sections. In part 1 of the study, an electronic learning (e-learning) module with a quality assessment program using the European Society of Pathology framework will be developed. This module will be used to assess the reliability and reproducibility of the TSR, conducted by national and international pathologists. Part 2 will involve the validation of the TSR in a prospective cohort of colon cancer p-stage II-III patients in a multicenter setting. In total, 1500 patients will be included.

Results: The results of part 1 will be expected in the first half of 2019. For part 2, the inclusion of patients in the prospective study, which started at the end of 2018, will take 3 years with an additional follow-up after another 3 years.

Conclusions: The main endpoints of this study are as follows: in part 1, trained (international) pathologists who are able to reliably score the TSR, resulting in low intra- and interobserver variation; in part 2, confirmation of significant survival differences for patients with a stroma-high tumor versus patients with a stroma-low tumor. On the basis of these findings, a modification in current treatment guidelines will be suggested.

Trial Registration: Netherlands Trial Register NTR7270; https://www.trialregister.nl/trial/7072

International Registered Report Identifier (IRRID): DERR1-10.2196/13464

(JMIR Res Protoc 2019;8(6):e13464) doi: 10.2196/13464 


\section{KEYWORDS}

tumor-stroma ratio; colon cancer; pathology; observer variation; prospective study

\section{Introduction}

\section{Background}

Despite complete resection of the primary tumor and surrounding lymph nodes, colon cancer patients often develop recurrence of disease, caused by the remaining micrometastases. These can be treated with chemotherapy. However, as micrometastases are difficult to detect, treatment guidelines are usually based on tumor characteristics related to disease progression and survival, such as depth of invasion and lymph node metastasis. The current guidelines advise to give adjuvant chemotherapy to patients with stage III colon carcinoma and patients with stage II and one or more high-risk factors [1]. Only a part of the patients who are treated with chemotherapy will actually benefit. Furthermore, there is also substantial undertreatment because $25 \%$ of the stage II patients, who do not receive adjuvant chemotherapy, will develop recurrence or metastasis within 5 years [2]. Some patients with stage IIIA receive adjuvant chemotherapy, whereas in some cases, the prognosis is better compared with patients with stage IIB disease [1]. The selection of colon cancer patients for adjuvant treatment should be further improved to establish an optimal treatment regimen for each patient.

Over the last decade, the microenvironment or stromal (ie, nonepithelial) component of tumors has been studied intensively. There is increasing evidence that the tumor stroma plays an important role in the biological behavior of tumors, their growth, ability to metastasize, but also their response or resistance to anticancer drugs [3-6]. Tumors that are rich in stroma behave in a more aggressive way compared with tumors with little stroma $[2,7]$.

\section{Tumor-Stroma Ratio}

The tumor-stroma ratio (TSR) parameter is based on the amount of stroma within the primary tumor and can be determined, without extra costs, during routine pathology assessment. Using the TSR, stage II/III stroma-high (high-risk) patients can be adequately registered for treatment with chemotherapy, whereas for the (elderly) patients with stage III and stroma-low, further discussion is needed as to whether adjuvant therapy will benefit these patients. New guidelines for patient management will have consequences for better patient management leading to a more optimal selection for adjuvant chemotherapy with a potential reduction in costs.

A high stroma percentage $(>50 \%)$ is an unfavorable prognostic factor. The TSR has been validated in various international studies with high interobserver agreements [2,7-12]. The TSR was discussed by the TNM Evaluation Committee, the Union for International Cancer Control, and the College of American Pathologists. They stated that our observations are important and novel and have the potential to be added to the TNM staging algorithm as prognosticator. They advocated validation in a prospective multicenter study and development of consensus agreement and a quality assessment program. This protocol elaborates on this recommendation.

\section{Objectives}

The overall objective is the addition of the TSR to current routine pathology next to the TNM classification for clinical decision making.

Primary objective for each part of the project:

1. Part 1: To evaluate and improve the reliability and reproducibility of pathologists specifically instructed for TSR scoring.

2. Part 2: To confirm the prognostic power of the method to select patients at risk for the development of recurrence of disease resulting in high-level evidence for adaptation of guidelines.

\section{Methods}

\section{Histopathological Scoring of the Tumor-Stroma Ratio}

For the evaluation of the TSR, Haematoxylin and Eosin (H\&E)-stained sections of the primary colon carcinoma, used in routine pathology to determine the T-stage (ie, the deepest part of the tumor), are analyzed using conventional microscopy. Areas with the largest amount of stroma are selected using a $2.5 \mathrm{x}$ or $5 \mathrm{x}$ objective. An area where both tumor and stromal tissue are present within this vision site is selected using a $10 \mathrm{x}$ objective. Tumor cells have to be present at all borders of the selected image field. Mucinous tumors, although more difficult, can also be scored; an area containing mucus may be used. However, the volume of mucus should be excluded when determining the TSR. Other challenging cases can be tumors with abundant necrosis and/or muscle tissue in between tumor glands. Necrotic areas or muscle tissue should be avoided in the scoring procedure. It is not necessary to score the TSR at the invasive front, picking a field with as much stroma as possible is more accurate.

Stroma-high is defined as $>50 \%$ stromal area and stroma-low, as $\leq 50 \%$ stromal area in the histological section (Figure 1). This cut-off has been determined a priori with maximum discriminative power [2,7,9]. Even if there is only one image field with a stroma-high score, this image field is decisive to categorize the patient as stroma-high.

The scoring protocol is available in an instruction movie on the Uniform Noting for International application of the Tumor-stroma ratio as Easy Diagnostic tool (UNITED) study website [13] and in written form published by our group [14]. 
Figure 1. Examples of stroma-low colon cancer (A) and stroma-high colon cancer (B).

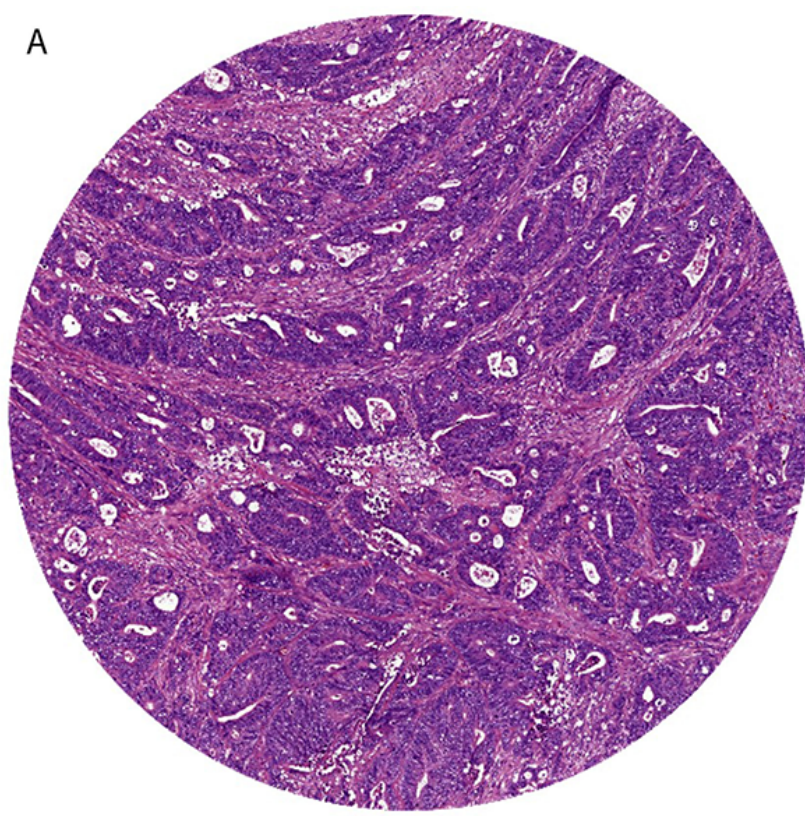

\section{Study Design}

Part 1 will consist of an electronic learning (e-learning) module which has been developed with a quality assessment program in the framework of the European Society of Pathology (ESP) External Quality Assessment program. Using this module, a reliability and reproducibility study on H\&E-stained tumor tissues will be conducted among national and international pathologists.

Part 2 will involve validation in a prospective cohort of colon cancer stage II-III patients within this multicenter setting. The inclusion is expected to take 3 years, with a 3 -year follow-up period.

\section{Patient Description}

In the UNITED study, all patients are diagnosed with pathological stage (p-stage) II or p-stage III colon cancer. For e-learning, H\&E-stained slides of stage II-III colon cancer patients were selected in a retrospective manner. Material was obtained from the archive of the Department of Pathology of the Leiden University Medical Center (LUMC).

\section{Part 1: The E-Learning Module}

An e-learning module has been developed in the framework of the ESP. The software used for the e-learning is PathXL Tutor version 6.1.1.1. (Philips). This is a Web-based software that can be accessed worldwide. Participating pathologists receive specific user credentials for access to the e-learning sets. The workflow is shown in Figure 2 and includes an introduction film with the technical instructions. Hereafter, the participating pathologists may start the e-learning by analyzing the training set containing 40 cases.

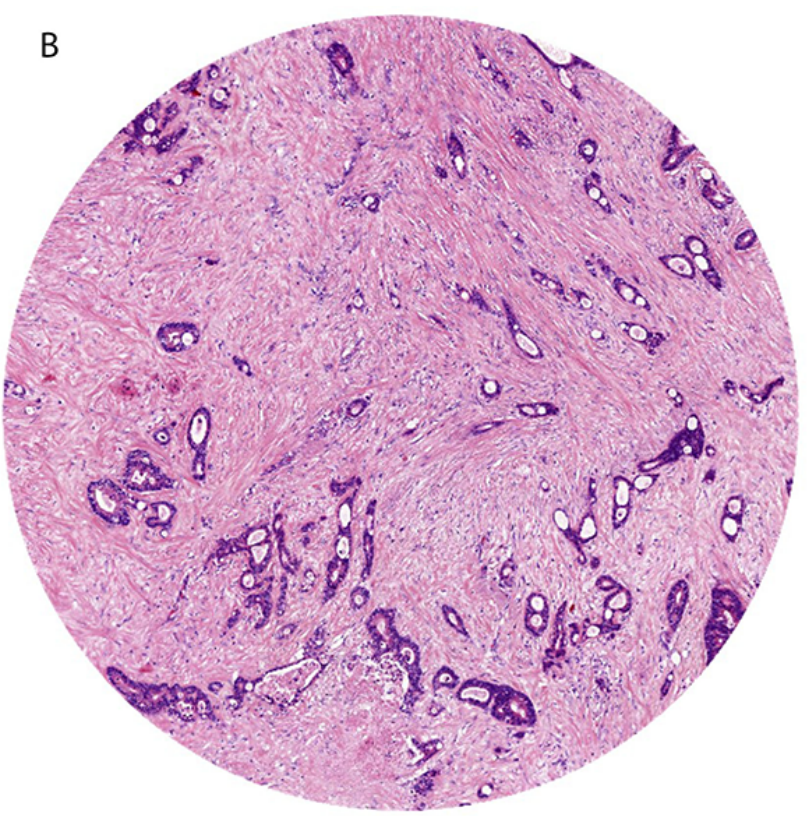

TSR scores of participating pathologists will be compared with a reference score (consisting of 3 observers at the LUMC). If a pathologist does not pass a set (interobserver variability kappa<.70), he or she is asked to re-analyze the set. If need be, the instruction video and protocol can be studied again. If a pathologist passes the set (kappa $\geq .70$ ), the pathologist is able to continue to the next set of 40 slides. The test set will be repeated after 2 months, thereafter inter- and intraobserver variability are determined. The pathologists are unaware of any clinical information or previous scoring.

The quality of TSR scoring by the participating pathologists will be monitored on a yearly basis by offering control series.

\section{Part 2: Validation of the Tumor-Stroma Ratio in a Prospective Study}

After finishing the e-learning, the pathologist is well instructed to score TSR in the daily routine. To validate the TSR prospectively, a multicenter study is set up. The study aims to include, in the participating centers, a total of 1500 colon cancer patients who have undergone complete curative resection (R0 resection), independent of receiving adjuvant chemotherapy according to actual guidelines.

\section{Recruitment of Patients and Consent}

Each consecutive eligible patient with a clinical stage I/II/III tumor will be informed about the study by their physician or research nurse. After informed consent, the pathologist is notified that the TSR can be determined. All patients, independent of gender and family history, are invited to participate. Medical history is no reason for exclusion, apart from malignancies within 10 years before the current colon carcinoma. Textboxes 1 and 2 describe the inclusion and exclusion criteria. 
Figure 2. Flowchart for the instruction of participating pathologists using the e-learning module.

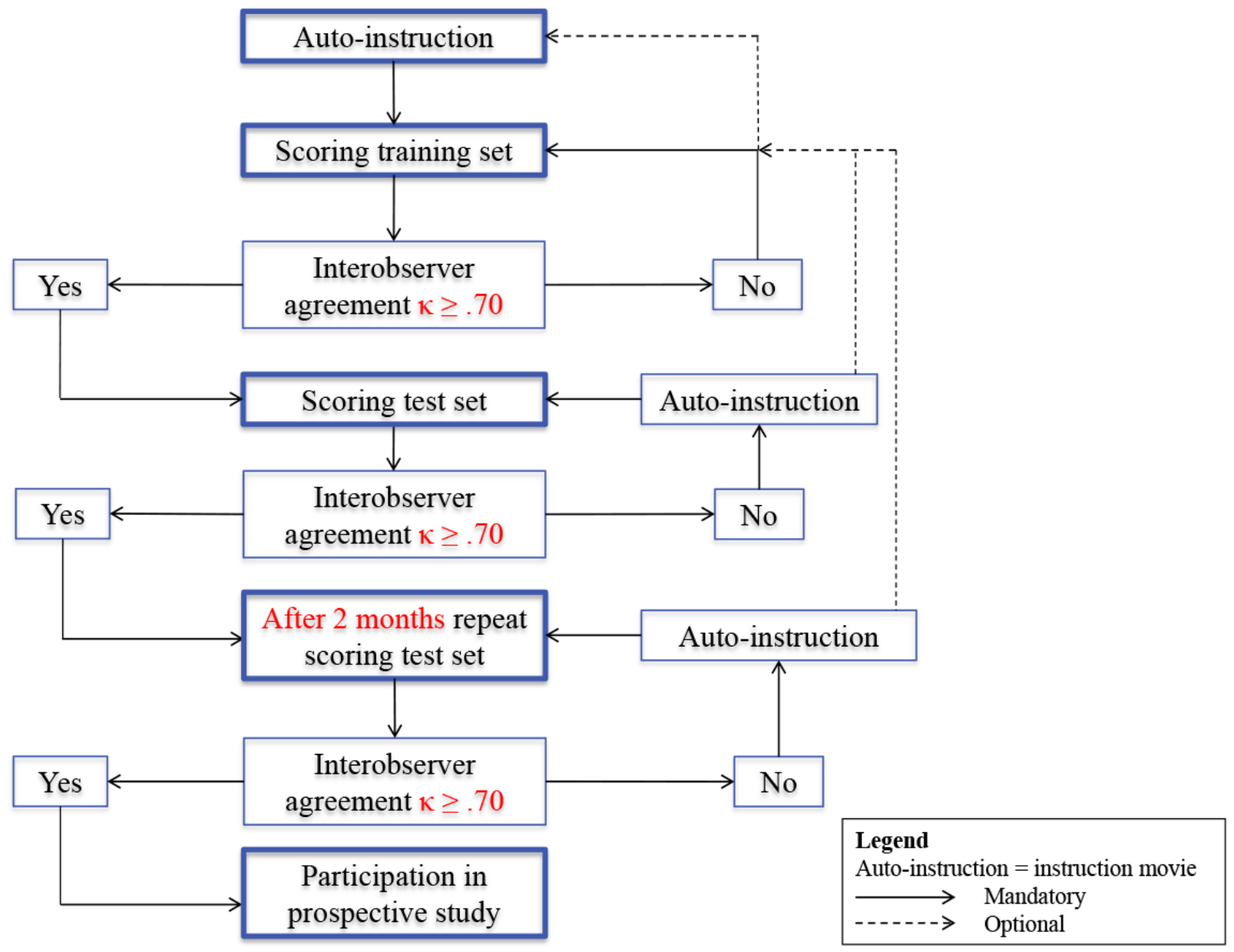

Textbox 1. Patient inclusion criteria.

- Histologically proven colon carcinoma

- $\quad$ Complete curative resection (R0 resection)

- $\quad$ Clinical stage I (T1-2, N0, M0), II (T3-4, N0, M0) or III (any T, N1-2, M0)

- $\quad$ Aged $\geq 18$ years

- Written informed consent

Textbox 2. Patient exclusion criteria.

- Neo-adjuvant treatment; this influences the amount of tumor and stroma, by fibrosis formation

- Any malignancy within 10 years before the current colon carcinoma (except for basal cell carcinoma or cervical carcinoma in situ) or any colon carcinoma in history; owing to prolonged treatment or metastasis from earlier primary tumors that can influence the current colon carcinoma prognosis. Basal cell carcinoma and cervical carcinoma in situ do not have metastatic capacity

- Multiple synchronous colon tumors; patients with synchronous tumors are likely to have a worse prognosis and need a different approach for treatment

- Rectum tumors; these are defined as separate entities. Prognosis and treatment is different compared with colon tumors

Additional exclusion after surgery:

- $\quad$-stage I or stage IV; p-stage I is excluded as these patients will not receive adjuvant treatment. Stage IV patients are excluded as these patients are palliatively treated

- Deceased within 3 months after surgery; patients who die within 3 months after surgery die most often owing to comorbidity or surgical complications 


\section{Safety Reporting and Risk Analysis}

The patient material to be analyzed in this study is a conventional H\&E-stained histological section of the primary tumor, obtained during the routine pathology process. The method is without any additional intervention and the study does not have consequences for the treatment of patients. Therefore, the safety or health of participating subjects will not be jeopardized in any way by this study. Consequently, no adverse events, serious adverse events, or suspected unexpected serious adverse events will occur owing to the study. A data safety monitoring board is not indicated.

\section{Data Storage}

The LUMC Datacenter, Department of Surgery, is the Central Datacenter and responsible for supply of electronic Case Report Forms, study database, generation of queries within the database, and central monitoring.

Data will be stored in Castor Electronic Data Capture (Castor EDC; Castor, Amsterdam, the Netherlands) [15]. Castor EDC is a cloud-based electronic data capture platform, easy-to-use by researchers worldwide and highly secured. Data can be easily captured; therefore, data are of high quality and reusable. Data and documents will be stored for at least 15 years.

\section{Statistical Analysis}

Statistical analysis will be performed using IBM SPSS Statistics version 25.0 in collaboration with the Department of Medical Statistics of the LUMC.

\section{Part 1}

For the analysis of the inter- and intraobserver variability, Cohen kappa coefficient will be used.

\section{Part 2}

\section{Sample Size Calculation}

For the prospective cohort, a sample size calculation has been performed for both stages based on earlier research findings $[2,7]$.

- $\quad$-stage II patients: with a hazard ratio (HR) of 2.0, adjusted for TNM, and a known percentage of stroma-high patients in p-stage II of $20 \%$ to $25 \%$ [2,7], 114 recurrence events with $90 \%$ power are necessary. To achieve 114 recurrence events based on an event rate of 0.0575 per year (leading to a 5-year probability of $75 \%$ and 3-year recurrence probability of $84.2 \%$ ), this leads to 722 patients.

- $\quad$-stage III patients: with an HR of 2.0, adjusted for TNM, and a known percentage of stroma-high patients in stage III of $30 \%$ to $35 \%$ [2,7], 97 recurrence events with $90 \%$ power are necessary. To achieve 97 recurrence events based on an event rate of 0.081 per year (leading to a 5-year probability of $66.7 \%$ and 3-year recurrence probability of $78.4 \%$ ), this leads to 450 patients.

To obtain 1172 evaluable p-stage II/III, approximately 1500 $(+25 \%)$ patients will be registered, as all p-stage I and stage IV patients will be excluded.

\section{Statistical Analysis}

Survival analysis will be performed using Kaplan-Meier survival analysis and differences in survival distributions will be tested using Log Rank statistics. The Cox proportional hazard model is used to determine the HR of explanatory variables for overall and disease-free survival (OS and DFS, respectively).

OS is defined as the time period between the date of surgery and the date of death from any cause or the date of the last follow-up. DFS is defined as the time between the date of surgery and the date of any recurrence (local, regional, or distant metastasis), date of new primary tumor, or date of death (any cause). If no event occurs, DFS is calculated as the time period until the date of last follow-up.

\section{Ethical Considerations}

This project is registered with the Netherlands Trial Registry (NTR 7270). It will be conducted according to the Declaration of Helsinki, Forteza, Brazil, October 2013.

As this research plan uses existing H\&E-stained sections, conventionally prepared for routine diagnostics, there is no risk for the patient, and we expect no problems with the regulatory authorities in the collaborating countries.

The UNITED study protocol has been approved by the Medical Research Ethics Committee (MREC) of the LUMC, study number p17.302. Before inclusion of patients in participating countries, the protocol will be endorsed by the MREC of each participating hospital.

Informed consent will be obtained from each eligible patient in oral and written form before surgery.

\section{Results}

\section{Part 1}

The e-learning started mid-2018 and the first results will be expected in the first half of 2019. The results will be presented within 6 to 12 months after the last pathologist has completed the e-learning module.

\section{Part 2}

The first patients were included at the end of 2018. In total, 1500 patients are needed, and the expected inclusion time is about 3 years. A follow-up of 3 years is required. In late 2023, the first results are expected, and they will be presented within 12 to 18 months after the last follow-up.

\section{Discussion}

The UNITED study has been developed to implement the TSR in routine pathology, in addition to the TNM classification and other known risk factors as an extra indicator for medical treatment decision making.

Earlier research validated the prognostic value of TSR in retrospective cohort studies. With the UNITED study, we aim to validate the prognostic value of the TSR in a prospective way. 
The results of the e-learning will contribute to a standardized method and specifically trained pathologists. With the yearly quality assessments, the quality of the scoring method will be monitored and guaranteed.

Beside the tumor characteristics, as described in the TNM classification, to determine the $\mathrm{p}$-stage, the microenvironment of the tumor is an important factor as well. The microenvironment of a tumor is a wide spread of different cell types. More tumor characteristics in the microenvironment are studied, such as tumor budding [16-21], Immunoscore [22-24], and desmoplastic reaction $[19,25,26]$. They are all independent prognostic biomarkers for survival [16-26]. Outside this protocol, we aim to study the relation between the different (microenvironment) biomarkers to better understand the role of the microenvironment and to further improve patient selection for adjuvant treatment.

Treatment decision making in oncology is a multidisciplinary process where medical oncologists play a pivotal role. These professionals will also be involved by the introduction of the TSR in daily clinical practice.

In conclusion, the UNITED study will, for the first time, evaluate the TSR in a prospective cohort to prove its prognostic value in stage II/III colon cancer. After completion of the UNITED study, the TSR will have the highest level of evidence for a prognostic marker and should be ready to use in the daily practice of all gastroenterology pathologists and also ready to play a role in clinical decision making.

\section{Acknowledgments}

The authors would like to thank all pathologists from the collaborating centers for participating in the UNITED study. The UNITED study is granted by the Dutch Cancer Society or KWF Kankerbestrijding (project 10174) and the Stichting Fonds Oncologie Holland.

\section{Conflicts of Interest}

None declared.

\section{References}

1. Labianca R, Nordlinger B, Beretta GD, Mosconi S, Mandalà M, Cervantes A, ESMO Guidelines Working Group. Early colon cancer: ESMO Clinical Practice Guidelines for diagnosis, treatment and follow-up. Ann Oncol 2013 Oct;24(Suppl 6):vi64-vi72. [doi: 10.1093/annonc/mdt354] [Medline: 24078664]

2. Mesker WE, Liefers G, Junggeburt JM, van Pelt GW, Alberici P, Kuppen PJ, et al. Presence of a high amount of stroma and downregulation of SMAD4 predict for worse survival for stage I-II colon cancer patients. Cell Oncol 2009;31(3):169-178 [FREE Full text] [doi: 10.3233/CLO-2009-0478] [Medline: 19478385]

3. Brown JM. Tumor microenvironment and the response to anticancer therapy. Cancer Biol Ther 2002;1(5):453-458. [Medline: 12496469]

4. Cukierman E, Khan DR. The benefits and challenges associated with the use of drug delivery systems in cancer therapy. Biochem Pharmacol 2010 Sep 1;80(5):762-770 [FREE Full text] [doi: 10.1016/j.bcp.2010.04.020] [Medline: 20417189]

5. Pouysségur J, Dayan F, Mazure NM. Hypoxia signalling in cancer and approaches to enforce tumour regression. Nature 2006 May 25;441(7092):437-443. [doi: 10.1038/nature04871] [Medline: 16724055]

6. Trédan O, Galmarini CM, Patel K, Tannock IF. Drug resistance and the solid tumor microenvironment. J Natl Cancer Inst 2007 Oct 3;99(19):1441-1454. [doi: 10.1093/jnci/djm135] [Medline: 17895480]

7. Mesker WE, Junggeburt JM, Szuhai K, de Heer P, Morreau H, Tanke HJ, et al. The carcinoma-stromal ratio of colon carcinoma is an independent factor for survival compared to lymph node status and tumor stage. Cell Oncol 2007;29(5):387-398 [FREE Full text] [Medline: 17726261]

8. West NP, Dattani M, McShane P, Hutchins G, Grabsch J, Mueller W, et al. The proportion of tumour cells is an independent predictor for survival in colorectal cancer patients. Br J Cancer 2010 May 11;102(10):1519-1523 [FREE Full text] [doi: 10.1038/sj.bjc.6605674] [Medline: 20407439]

9. Huijbers A, Tollenaar RA, van Pelt GW, Zeestraten EC, Dutton S, McConkey CC, et al. The proportion of tumor-stroma as a strong prognosticator for stage II and III colon cancer patients: validation in the VICTOR trial. Ann Oncol 2013 Jan;24(1):179-185. [doi: 10.1093/annonc/mds246] [Medline: 22865778]

10. Park JH, Richards CH, McMillan DC, Horgan PG, Roxburgh CS. The relationship between tumour stroma percentage, the tumour microenvironment and survival in patients with primary operable colorectal cancer. Ann Oncol 2014 Mar;25(3):644-651 [FREE Full text] [doi: 10.1093/annonc/mdt593] [Medline: 24458470]

11. van Pelt GW, Hansen TF, Bastiaannet E, Frifeldt SK, van Krieken JH, AEM Tollenaar R, et al. Stroma-high lymph node involvement predicts poor survival more accurately for patients with stage III colon cancer. J Med Surg Pathol 2016;01(02). [doi: 10.4172/2472-4971.1000116]

12. Hynes SO, Coleman HG, Kelly PJ, Irwin S, O'Neill RF, Gray RT, et al. Back to the future: routine morphological assessment of the tumour microenvironment is prognostic in stage II/III colon cancer in a large population-based study. Histopathology 2017 Jul;71(1):12-26. [doi: 10.1111/his.13181] [Medline: 28165633] 
13. Smit MA, van Pelt GW, Tollenaar RA, Mesker WE. Watch Stroma. Uniform Noting for International application of the Tumour-stroma ratio as Easy Diagnostic Tool (UNITED) study website URL: http://watchstroma.com/ [accessed 2019-05-02] [WebCite Cache ID 784El3ZGH]

14. van Pelt GW, Kjær-Frifeldt S, van Krieken JH, Al Dieri R, Morreau H, Tollenaar RA, et al. Scoring the tumor-stroma ratio in colon cancer: procedure and recommendations. Virchows Arch 2018 Oct;473(4):405-412 [FREE Full text] [doi: 10.1007/s00428-018-2408-z] [Medline: 30030621]

15. Castor EDC. URL: https://www.castoredc.com/ [accessed 2019-05-06] [WebCite Cache ID 78A3ksBYx]

16. Nakamura T, Mitomi H, Kanazawa H, Ohkura Y, Watanabe M. Tumor budding as an index to identify high-risk patients with stage II colon cancer. Dis Colon Rectum 2008 May;51(5):568-572. [doi: 10.1007/s10350-008-9192-9] [Medline: 18286339]

17. Betge J, Kornprat P, Pollheimer MJ, Lindtner RA, Schlemmer A, Rehak P, et al. Tumor budding is an independent predictor of outcome in AJCC/UICC stage II colorectal cancer. Ann Surg Oncol 2012 Nov;19(12):3706-3712. [doi: 10.1245/s10434-012-2426-z] [Medline: 22669453]

18. Betge J, Langner C. Vascular invasion, perineural invasion, and tumour budding: predictors of outcome in colorectal cancer. Acta Gastroenterol Belg 2011 Dec;74(4):516-529. [Medline: 22319961]

19. Ueno H, Shinto E, Shimazaki H, Kajiwara Y, Sueyama T, Yamamoto J, et al. Histologic categorization of desmoplastic reaction: its relevance to the colorectal cancer microenvironment and prognosis. Ann Surg Oncol 2015 May;22(5):1504-1512. [doi: 10.1245/s10434-014-4149-9] [Medline: 25395146]

20. van Wyk HC, Park JH, Edwards J, Horgan PG, McMillan DC, Going JJ. The relationship between tumour budding, the tumour microenvironment and survival in patients with primary operable colorectal cancer. Br J Cancer 2016 Dec 12;115(2):156-163 [FREE Full text] [doi: 10.1038/bjc.2016.173] [Medline: 27299960]

21. Eriksen AC, Sørensen FB, Lindebjerg J, Hager H, dePont Christensen R, Kjær-Frifeldt S, et al. The prognostic value of tumour stroma ratio and tumour budding in stage II colon cancer. A nationwide population-based study. Int J Colorectal Dis 2018 Aug;33(8):1115-1124 [FREE Full text] [doi: 10.1007/s00384-018-3076-9] [Medline: 29785462]

22. Galon J, Costes A, Sanchez-Cabo F, Kirilovsky A, Mlecnik B, Lagorce-Pagès C, et al. Type, density, and location of immune cells within human colorectal tumors predict clinical outcome. Science 2006 Sep 29;313(5795):1960-1964 [FREE Full text] [doi: 10.1126/science.1129139] [Medline: 17008531]

23. Angell H, Galon J. From the immune contexture to the Immunoscore: the role of prognostic and predictive immune markers in cancer. Curr Opin Immunol 2013 Apr;25(2):261-267. [doi: 10.1016/j.coi.2013.03.004] [Medline: 23579076]

24. Pagès F, Mlecnik B, Marliot F, Bindea G, Ou F, Bifulco C, et al. International validation of the consensus Immunoscore for the classification of colon cancer: a prognostic and accuracy study. Lancet 2018 Dec 26;391(10135):2128-2139. [doi: 10.1016/S0140-6736(18)30789-X] [Medline: 29754777]

25. Ueno H, Kanemitsu Y, Sekine S, Ishiguro M, Ito E, Hashiguchi Y, et al. Desmoplastic pattern at the tumor front defines poor-prognosis subtypes of colorectal cancer. Am J Surg Pathol 2017 Nov;41(11):1506-1512. [doi:

10.1097/PAS.0000000000000946] [Medline: 28877064]

26. Ueno H, Sekine S, Oshiro T, Kanemitsu Y, Hamaguchi T, Shida D, et al. Disentangling the prognostic heterogeneity of stage III colorectal cancer through histologic stromal categorization. Surgery 2018 Dec;163(4):777-783. [doi: 10.1016/j.surg.2017.09.007] [Medline: 29162344]

\section{Abbreviations}

Castor EDC: Castor Electronic Data Capture

DFS: disease-free survival

e-learning: electronic learning

ESP: European Society of Pathology

H\&E: Haematoxylin and Eosin

HR: hazard ratio

LUMC: Leiden University Medical Center

MREC: Medical Research Ethics Committee

OS: overall survival

p-stage: pathological stage

TNM: Tumor-Node-Metastasis

TSR: tumor-stroma ratio

UNITED: Uniform Noting for International application of the Tumor-stroma ratio as Easy Diagnostic tool 
Edited by G Eysenbach; submitted 23.01.19; peer-reviewed by MS Aslam, P Muddegowda; comments to author 28.03.19; revised version received 13.05.19; accepted 14.05.19; published 14.06.19

Please cite as:

Smit M, van Pelt G, Roodvoets A, Meershoek-Klein Kranenbarg E, Putter H, Tollenaar R, van Krieken JH, Mesker W

Uniform Noting for International Application of the Tumor-Stroma Ratio as an Easy Diagnostic Tool: Protocol for a Multicenter

Prospective Cohort Study

JMIR Res Protoc 2019;8(6):e13464

URL: http://www.researchprotocols.org/2019/6/e13464/

doi: $\underline{10.2196 / 13464}$

PMID: $\underline{31199317}$

CMarloes Smit, Gabi van Pelt, Annet Roodvoets, Elma Meershoek-Klein Kranenbarg, Hein Putter, Rob Tollenaar, J Han van Krieken, Wilma Mesker. Originally published in JMIR Research Protocols (http://www.researchprotocols.org), 14.06.2019. This is an open-access article distributed under the terms of the Creative Commons Attribution License (https://creativecommons.org/licenses/by/4.0/), which permits unrestricted use, distribution, and reproduction in any medium, provided the original work, first published in JMIR Research Protocols, is properly cited. The complete bibliographic information, a link to the original publication on http://www.researchprotocols.org, as well as this copyright and license information must be included. 